\title{
Situative (Nicht-)Passung als Erklärungsansatz von Drop-out in der Weiterbildung
}

\author{
Stefanie Hoffmann (D) - Veronika Thalhammer • Aiga von Hippel • \\ Bernhard Schmidt-Hertha
}

Eingegangen: 27. Mai 2021 / Überarbeitet: 20. September 2021 / Angenommen: 25. Oktober 2021 / Online publiziert: 16 . November 2021

(C) Der/die Autor(en) 2021

Zusammenfassung Trotz der Vielzahl seiner Erscheinungs- und Umgangsformen findet Drop-out in der erwachsenenpädagogischen Forschung vergleichsweise wenig Beachtung und theoretische Konzepte, welche die unterschiedlichen Lesarten des Phänomens in einem übergeordneten Rahmen systematisieren, gibt es bislang kaum. Im Beitrag werden daher bisherige theoretische Konzepte zu Weiterbildungsabbrüchen und Ansätze zur Einordnung von Weiterbildungsabbrüchen als Weiterbildungsverhalten zusammengetragen sowie die Forschungslücken in Bezug auf ein umfassendes Modell zur ursächlichen Erklärung von Drop-out in der Weiterbildung aufgezeigt. Anhand einer empirischen Studie - hier basierend auf der Auswertung von 40 problemzentrierten Interviews - werden eine Typologie sowie ein Modell zur Charakterisierung von Weiterbildungsabbrüchen vorgeschlagen und deren Potenzial für die weitere Theorieentwicklung in diesem Bereich herausgearbeitet. Als zentral kristallisiert sich dabei der Begriff „Passung“ heraus, mit dem sich die Vielfalt von Abbruchskonstellationen theoretisch anschlussfähig beschreiben lässt.

Schlüsselwörter Weiterbildungsverhalten · Drop-out · Passung · Typologie · Modell · Weiterbildungsabbruch

Stefanie Hoffmann, M.A. ( $\varangle) \cdot$ Prof. Dr. Aiga von Hippel

Humboldt-Universität zu Berlin, Berlin, Deutschland

E-Mail: s.hoffmann@hu-berlin.de

Prof. Dr. Aiga von Hippel

E-Mail: aiga.von.hippel@hu-berlin.de

Dr. Veronika Thalhammer · Prof. Dr. Bernhard Schmidt-Hertha

Ludwig-Maximilians-Universität München, München, Deutschland

Dr. Veronika Thalhammer

E-Mail: veronika.thalhammer@1mu.de

Prof. Dr. Bernhard Schmidt-Hertha

E-Mail: b.schmidt@edu.lmu.de 


\section{Situational (non-)fit as an explanatory approach to dropout in further education}

Abstract Despite the large number of its different forms of manifestation and of handling, dropout has so far received comparatively little attention in research on adult education. There is only a small number of theoretical concepts that systematize the diverse interpretations of the phenomenon within a superordinate framework. This study aims at contributing to that question. To that end, theoretical concepts of dropout in further education and approaches to an interpretation of dropout from further education as a further-education-specific behavior are collated and the research gaps with regard to a comprehensive model explaining the reasons for dropping out from further education are revealed. By referring to an empirical study-based on the evaluation of 40 problem-centered interviews - a typology as well as a model for the characterization of dropout cases is proposed and its potential for further theory building in this field is explored. Through this approach, the central concept of "fit" emerges, which allows to describe the variety of dropout constellations in a theoretically compatible way.

Keywords Further education behavior · Dropout · Fit · Typology · Model

\section{Einleitung}

Der Abbruch von Weiterbildungsteilnahmen gehört einerseits zum Alltag erwachsenenpädagogischer Praxis und findet andererseits vergleichsweise wenig Beachtung in wissenschaftlichen Arbeiten. Dabei kann die vorzeitige Beendigung einer Teilnahme sehr unterschiedlich interpretiert werden, z. B. als rationale Entscheidung im Kontext sich verändernder individueller Lebenswelten, als Erreichen eines selbstgesteckten Bildungsziels, als Scheitern erwachsenenpädagogischer Bemühungen. Theoretische Konzepte, die die unterschiedlichen Lesarten in einem übergeordneten Rahmen systematisieren, gibt es bislang kaum. Es stellt sich also die Frage nach Ursachen und Bedingungen von Drop-outs in der Weiterbildung, zu deren Beantwortung dieser Artikel einen Beitrag leisten will. Als zentral kristallisiert sich dabei der Begriff der Passung heraus, mit dem sich die Vielfalt von Abbruchursachen theoretisch anschlussfähig beschreiben lässt (Abschn. 4.1). Im Folgenden werden bisherige theoretische Konzepte und Ansätze zur Einordnung von Weiterbildungsabbrüchen gesichtet und nach deren empirischer Bewährung gefragt (Abschn. 2). Anhand einer empirischen Studie (Abschn. 3) werden dann eine Typologie und ein Modell zur Charakterisierung von Weiterbildungsabbrüchen vorgeschlagen und deren Potenzial für die weitere Theorieentwicklung in diesem Bereich herausgearbeitet (Abschn. 4 und 5). 


\section{Drop-out in theoretischen und empirischen Perspektiven}

Anknüpfend an die bereits publizierten Ergebnisse aus den ersten theoretischen und empirischen Vorarbeiten der Gruppe von Autorinnen und Autoren (siehe Hoffmann et al. 2020) wird im Folgenden das begriffliche Verständnis von Drop-out präzisiert und ein grober Überblick über den Forschungsstand zu Drop-out in der Erwachsenenbildung gegeben.

\subsection{Das Phänomen Drop-out}

Kursteilnahmen können als subjektiv bedeutsame Statuspassagen gelten. Dies wird besonders deutlich, wenn durch den Drop-out ein eigentlich vorhandenes Weiterbildungsinteresse nicht weiter realisiert wird und/oder damit ein grundsätzlicher Rückzug aus dem System Weiterbildung eingeleitet wird (vgl. Schmidt 2011). Das antonymische Phänomen der Kursbindung steht hierzu in Kontrast: in Form eines dauerhaften Verharrens von Teilnehmerinnen und Teilnehmern in Kursen (bedingt beispielsweise durch die Schaffung neuer Abhängigkeiten), wie u. a. aus dem Bereich der Alphabetisierung und Grundbildung bekannt (vgl. Egloff et al. 2009, S. 17).

Das explizit auf Drop-out in der Weiterbildung bezogene psychologisch fundierte Kongruenzmodell von Boshier (1973) konzentriert sich auf das Zusammenspiel zwischen Lernenden und Lernumgebung und das Selbstkonzept des Lernenden (v. a. in Bezug auf dessen Mangel- und Wachstumsorientierung). Geleitet vom Streben des Individuums nach Aufrechterhaltung der inneren Harmonie zwischen sich selbst und dem Lernumfeld (v.a. hinsichtlich Dozentinnen und Dozenten sowie anderen Teilnehmenden) stimmen im Idealfall das Erleben des Selbst mit dem idealen Selbstkonzept überein (Kongruenz). Entscheidend für den Verbleib oder Drop-out sei letztendlich der Grad der Übereinstimmung bzw. des Gleichgewichts.

Verwiesen wird zudem häufig auf das aus der Hochschulforschung stammende Integrationsmodell von Tinto $(1975,1993)$, das aus einem institutionsorientierten Blickwinkel heraus die Bedeutung der akademischen sowie der sozialen Integration und die Einflussnahme verschiedener Faktoren auf die Entwicklung des Drop-outs $\mathrm{zu}$ verschiedenen Zeitpunkten des Bildungsprozesses betont. Ob sich ein Drop-out vollziehe, sei letztendlich nicht nur vom Commitment zur Hochschule abhängig, sondern auch von der Balance zu außerinstitutionellen Commitments und den jeweilig spezifischen Werten und Verhaltensnormen der unterschiedlichen Gemeinschaften.

Weiterbildungsbeteiligung ist in dem hier zugrundliegenden Verständnis ,nur als subjektiv begründetes Handeln von Akteuren innerhalb von gesellschaftlichen Strukturen zu begreifen“ (Käpplinger et al. 2013, S. 18). Vor diesem Hintergrund ist auch der Drop-out-Prozess nicht nur von subjektbezogenen Faktoren (z. B. persönliche Erfahrungen, Fähigkeiten und Einstellung), sondern von unzählig vielen, miteinander verflochtenen Faktoren (wie z. B. geografische, soziale, kulturelle, zeitliche oder wirtschaftliche Faktoren des Kurses oder der Institution) abhängig und infolgedessen kaum verallgemeinerbar (vgl. d. Carmo Nicoletti 2019). So erfordern die besonderen Bedingungen im quartären Bildungssektor (z. B. größtenteils Freiwilligkeit der Teilnahme, non-formaler Charakter, Programmplanung statt festes Curriculum etc.) eine eigenständige Erklärung des Phänomens. 
In dem diesem Beitrag zugrundeliegenden Forschungsprojekt (siehe Abschn. 3) wird Drop-out als Teil des Weiterbildungsverhaltens betrachtet, das - wie die Weiterbildungsbeteiligung insgesamt - als multifaktoriell bedingt $\mathrm{zu}$ verstehen ist (vgl. Reich-Claassen 2015). Nicht Einzelmotive sind dabei für Entscheidungen ausschlaggebend, sondern divergente Motivationen (vgl. Reich-Claassen 2010), Interessengemengelagen (vgl. Grotlüschen 2010) und Emotionen (vgl. Gieseke 2016) wirken interdependent. Die Bedeutungszumessung von Drop-out ist auch nicht nur von den konkreten Erwartungshaltungen hinsichtlich des Veranstaltungsbesuchs und der Lebensperspektiven der Teilnehmenden abhängig zu machen, sondern auch von der ,gesellschaftlich bedingten Behinderungen einer kontinuierlichen bzw. gescheiterten Veranstaltungsteilnahme im Erwachsenenalter" (Brödel 1996, S. 23). Unter Berücksichtigung der „Motivverkopplung“ (Vontobel 1972) sind Ursachen von Dropouts dann weniger in Entweder-Oder-Zuschreibungen zu finden, sondern in einem Spannungsverhältnis zwischen divergenten individuellen, situativen, institutionellen sowie (sozio-)strukturellen Aspekten.

\subsection{Forschungsstand}

Im deutschsprachigen Raum wurden nach einigen empirischen Untersuchungen auf Basis von Teilnehmendenstatistiken und -befragungen in den 1970er (im Überblick bei Nuissl und Sutter 1979) und 1980er Jahren (z. B. Gruber 1985; Schrader 1986) Abbrüche bzw. Drop-outs in der Erwachsenenbildung kaum mehr thematisiert. Danach finden sich nur noch sehr wenige empirische Studien zu diesem Thema, die sich vor allem auf das Phänomen Drop-out selbst bzw. dessen Prävalenz konzentrieren. Ausnahmen dazu stellen im deutschsprachigen Raum z. B. die Untersuchungen zu Drop-outs in Funkkolleg-Begleitkursen (Lingkost 1996) oder Studien im Bereich der Grundbildung dar (Egloff et al. 2009; Egloff 2011; Jakschik und Pieniazeka 2011), die jeweils bereichsspezifisch und nur bedingt verallgemeinerbar sind. Das ist insofern von besonderer Bedeutung, da Analysen auf Basis repräsentativer Weiterbildungsstudien (Schmidt 2011; Hoffmann et al. 2020) darauf hindeuten, dass sich Abbrüche hinsichtlich ihrer Hintergründe und Kontexte stark unterscheiden und sowohl bei sehr weiterbildungsaktiven Personen als auch bei eher bildungsfernen Zielgruppen vorkommen.

Das Feld der internationalen Forschung zu Abbrüchen in der Erwachsenenbildung zeichnet sich durch ein breites Spektrum analytischer Perspektiven aus, das sowohl unterschiedliche Teilnehmendengruppen oder Forschungssettings als auch spezielle Kursformate umfasst (z. B. Bariso 2008; Park und Choi 2009). Es liegen zahlreiche Studien zu Studienabbrüchen vor, einschließlich einiger Arbeiten zum Verbleib Studierender (vgl. McGivney 2004). Die qualitative Studie von Jacot et al. (2010) weist auf die Relevanz von konkurrierenden Lebensbereichen hin, die für einen Studienabbruch verantwortlich sein können. Eine frühere quantitative Untersuchung von Shank und McCracken (1993) über Abbrechende in der beruflichen Erwachsenenbildung unterstreicht Fragen der finanziellen Unterstützung sowie der sozialen und akademischen Integration im Kontext von Weiterbildungsabbrüchen. Darüber hinaus werden soziale Prozesse innerhalb der Lernveranstaltungen für Abbrüche verantwortlich gemacht (z. B. Ten Dam 1995). 
In der Weiterbildung kann ein Abbruch ganz unterschiedliche Bedeutungen und Hintergründe haben. Da viele Programme über einen längeren Zeitraum gehen, ist die Wahrscheinlichkeit hoch, dass sich in dieser Zeit signifikante Veränderungen in anderen Lebensbereichen ergeben und die persönlichen Lernziele könnten lange vor offiziellem Ende des Kurses erreicht werden (vgl. Hoffmann et al. 2020; Pickard 2013).

Zusammenfassend lässt sich sagen, dass der Forschungsstand zwei Schwerpunkte erkennen lässt: Zum einen werden Merkmale der Bildungsbeteiligung durch quantitative Erhebungen zu soziologischen Merkmalen oder qualitativ durch den Zugang zu Bildungsbiographien untersucht. Zum anderen werden die Organisationen der Erwachsenenbildung selbst im sozialen und organisatorischen Kontext in den Blick genommen (vgl. Bourgeois 1998, S. 333). Betrachtet man diese Studien im Detail, so zeigt sich, dass eine Herausforderung für zukünftige Untersuchungen und für die Theoriebildung in der Verbindung des Individuums mit der Institution zu liegen scheint (vgl. Jacot et al. 2010).

Was daher auch benötigt wird, ist ein inklusives theoretisches Modell zur Analyse des Abbruchs in verschiedenen Kontexten der Erwachsenenbildung (vgl. auch Garrison 1987). An dieses Forschungsdefizit knüpft die Studie an, die im Folgenden in ihrer methodischen Anlage vorgestellt wird.

\section{Eigene empirische Zugänge zur Erforschung von Drop-out}

Die Ergebnisse des vorliegenden Beitrags wurden im DFG-geförderten Forschungsprojekt „Analyse von Drop-out in der Weiterbildung“ erarbeitet. Die übergeordneten Fragestellungen waren (1) in welchen Konstellationen es zu Drop-out kommt und (2) welche Gründe als ausschlaggebend für Drop-out gesehen werden. Grundannahme des Projekts war das Zusammenspiel von (sozio-)strukturellen, institutionellen, individuellen und situativen Dimensionen bei Drop-out-Prozessen.

Um unterschiedliche Perspektiven zu erschließen, wurden drei verschiedene $\mathrm{Zu}$ gänge kombiniert und damit drei Teilstudien durchgeführt: Zunächst wurden Daten aus der Erwachsenenkohorte des Nationalen Bildungspanels (NEPS) zur Identifizierung von Prädiktoren für Weiterbildungsabbrüche analysiert. Parallel wurden bundesweit elf Interviews mit Expertinnen und Experten (vgl. Meuser und Nagel 2002) aus verschiedenen Weiterbildungsverbänden geführt (vgl. Hoffmann et al. 2020). In einem dritten Zugang wurden 40 problemzentrierte Interviews (vgl. Witzel 2000) mit Personen geführt, die ihre Weiterbildung abbrachen, um subjektive Bedeutsamkeit und Gründe von Drop-out, Verläufe und Dynamiken der Drop-out-Erfahrungen im Zusammenspiel der verschiedenen Faktoren herauszuarbeiten: Diese Interviews liegen den in diesem Beitrag vorgestellten Analyseergebnissen zugrunde.

\subsection{Datenerhebung und Sampling: Interviews mit weiterbildungsabbrechenden Personen}

Mittels der 40 problemzentrierten Interviews (PZI) (vgl. Witzel 2000) mit Personen, die ihre Weiterbildung abbrachen, wurden die Abbruchserfahrungen der Weiterbil- 
dungsteilnehmenden und die individuellen Wahrnehmungs- und Umgangsweisen der (ehemaligen) Weiterbildungsteilnehmenden mit der Drop-out-Erfahrung erfasst. Die Teilstudie orientierte sich an den übergeordneten Fragestellungen der Gesamtstudie, stellte jedoch die individuellen Deutungen und Erfahrungen hinsichtlich der Entstehung und Begründung von Drop-out aus der Perspektive der befragten Personen in den Fokus.

Die Auswahl der Personen und die Leitfadenkonstruktion orientierten sich an den Ergebnissen der quantitativen Analyse sowie an den Ergebnissen der Interviews mit den Expertinnen und Experten. Das PZI (vgl. Witzel 2000) ermöglichte durch das in der Methode angelegte induktiv-deduktive Wechselspiel eine möglichst unvoreingenommene Erfassung und Auswertung individueller Handlungen, subjektiver Wahrnehmungen und Verarbeitungsweisen hinsichtlich der gemachten Drop-out-Erfahrung. So wurden die Interviewpartnerinnen und -partner durch die Leitfragen zu Narrationen angeregt, die sich an dem Problemgegenstand orientierten, und durch Nachfragen im Dialogverfahren wurde eine befragtenzentrierte Kommunikationssituation erzeugt. Im Zentrum der Interviews stand die Frage nach der Vorgeschichte des Weiterbildungsabbruchs, darüber hinaus wurden auch situationsbezogene (z. B. berufliche und familiäre Hintergründe), individuelle (z. B. bildungsbiographisch verankerte Dispositionen und Bildungseinstellungen), strukturelle (z. B. ökonomische und soziale Rahmenbedingungen) sowie institutionelle und angebotsbezogene Merkmale der Weiterbildung (z. B. hinsichtlich der Themen, Dozentinnen und Dozenten und des Trägers) erfragt. In den Interviews wurden somit Rahmenbedingungen, Genese und insbesondere individuell handlungsleitende Motive von Weiterbildungsabbrüchen im Dialog herausgearbeitet, aber auch die retrospektive Bewertung der eigenen Entscheidung und der daraus resultierenden Folgen thematisiert. Standardisierte Fragen, etwa zum Weiterbildungsangebot und zur Bildungsbiographie, wurden mittels eines ergänzenden Kurzfragebogens nach dem Interview beantwortet. Die Interviews wurden aufgezeichnet und vollständig transkribiert. Nach den Gesprächen wurde die Besonderheit der jeweiligen Gesprächssituation durch Postskripte festgehalten.

Beim Sampling der 40 Studienteilnehmenden wurden Elemente des kriteriengeleiteten Samplings mit Zielen eines eher theoretischen bzw. theoriegenerierenden Samplings einbezogen (vgl. Przyborski und Wohlrab-Sahr 2014). Es zielte auf eine Variation und Intensität der Verdichtung/Heterogenität im Sample auf Ebene der Konstellationen von Drop-out (vgl. Flick 2007, S. 165; Merkens 1997). Die Auswahl der Interviewpartnerinnen und -partner erfolgte somit zum Teil nach dem theoretical sampling (vgl. Strauss und Corbin 1996), aber auch - abweichend von einem strengen Verständnis des theoretical sampling - aufgrund von plausiblen Vorannahmen (u. a. in Bezug auf Weiterbildungsart, Geschlecht, Weiterbildungsteilnahme) bezugnehmend auf vorhandene theoretische und empirische Studien (vgl. u.a. ReichClaassen 2010; Schmidt 2011).

Berücksichtigt wurden somit bereits vorliegende Erkenntnisse zu den mit Dropout zusammenhängenden Konstellationen (z. B. bzgl. Dauer und Finanzierung der Weiterbildung) aus den anderen methodischen Zugängen (Interviews mit Expertinnen und Experten und NEPS-Analysen) innerhalb des Forschungsprojektes (vgl. Hoffmann et al. 2020). Auch weil unterschiedliche Interessefokusse und die un- 
terschiedlichen Verbindlichkeitsgrade differente Entscheidungsprozesse hinsichtlich Verbleib und Abbruch erwarten ließen, wurden beispielsweise auf intentioneller Ebene gezielt Drop-out-Erfahrungen von Abbrecherinnen und Abbrechern in beruflichen und allgemeinen sowie in non-formalen und formalen Weiterbildungsangeboten einbezogen. Hinsichtlich des Alters (Variation im Sample zwischen 24 und 68 Jahren) der Teilnehmenden wurde auf eine möglichst große Bandbreite geachtet, damit auch alternsbedingte/gesundheitliche Drop-out-Gründe nicht außer Acht gelassen wurden. Gezielt wurden neben den 29 weiblichen Interviewteilnehmerinnen elf männliche Personen mit Drop-out-Erfahrung interviewt. Hinsichtlich des höchsten Schulabschlusses wurde darauf geachtet, dass nicht nur Personen mit hohem Bildungsabschluss in die Stichprobe einbezogen werden: 35 Personen haben einen höheren Schulabschluss, drei haben einen Realschulabschluss, eine Person hat einen Hauptschulabschluss und eine hat die Schule abgebrochen. Auf diese Weise wurde eine möglichst kontrast- und variationsreiche Stichprobe generiert, die Drop-out-Erfahrungen der Teilnehmenden in sehr unterschiedlichen Weiterbildungen abbildet: Es gibt deutliche Variationen in der Dauer (zwischen zwei Wochen und mehreren Jahren), aber auch hinsichtlich der thematischen Inhalte und Zielsetzungen der erfassten Weiterbildungen, der Gruppengrößen und der Finanzierungsformen.

In verschiedenen Einrichtungen der Erwachsenenbildung und weiteren, bewusst breit gestreuten, öffentlich zugänglichen Orten (z. B. öffentliche Bibliotheken, Jobcenter, Volkshochschulen, Einrichtungen der Lernberatung, Seniorentreffs, Aushangmöglichkeiten in Bäckereien und Supermärkten) wurde auf die Studie aufmerksam und damit die Möglichkeit der freiwilligen Teilnahme an Interviews publik gemacht. Parallel zur Analyse der Interviewdaten wurde bei dieser Strategie des Feldzugangs bewusst eine hohe Flexibilität beibehalten und die Zugangsorte und Kommunikationsmedien (z. B. E-Mail-Verteiler) kontinuierlich erweitert. Durch dieses Vorgehen lagen ca. 80 Meldungen von potenziellen Interviewteilnehmerinnen und -teilnehmern vor, sodass das Sampling auf die Analyse folgen konnte und die Analyse jeweils die weitere Datensammlung und die Erweiterung des Feldzugangs leitete (vgl. Strauss und Corbin 1996).

Als Voraussetzung für die Interviewteilnahme galt das Vorliegen mindestens einer Weiterbildungs-Drop-out-Erfahrung, welche durch ein telefonisches Vorgespräch ermittelt und ggf. im persönlich und in Präsenz durchgeführten Interview ausführlich thematisiert wurde. So haben alle Interviewteilnehmenden trotz der großen Varianz im Sampling eines gemeinsam: Sie haben mindestens eine oder mehrere Abbruchserfahrungen in der Erwachsenenbildung gemacht, auch wenn diese Vorkommnisse in ihrer Erscheinungsform und hinsichtlich des Umgangs der Individuen mit den Abbruchserfahrungen variieren. Die Auswahl der Studienteilnehmenden erfolgte somit auch anhand der im Vorgespräch skizzierten Abbruchserfahrungen und damit nach dem theoretischen Sampling (vgl. Strauss und Corbin 1996), ,auf Basis von Konzepten, die eine bestätigte theoretische Relevanz für die sich entwickelnde Theorie besitzen“ (vgl. ebd., S. 148). 


\subsection{Analyse der Interviews}

Bei der Analyse der Interviews mit den Abbrecherinnen und Abbrechern kamen methodische Techniken zum Einsatz, die auf Prämissen der Grounded Theory (vgl. Strauss und Corbin 1996) basieren. So wurde z. B. jede im Interview empirisch ermittelte Abbruchserfahrung unter Bezugnahme auf das Kodierparadigma (ebd., S. 78) in der jeweils eigenen Komplexität systematisiert und visualisiert. Damit wurden u.a. tiefergehende Ansätze zu Handlungsmotiven und Interpretationsschemata der Befragten ergründet, die deutlich über die explizit geäußerten Abbruchsgründe hinausgehen. Auf diese Weise konnten durch die Analyse des Datenkorpus insgesamt 48 individuelle Abbruchserfahrungen in Weiterbildungen in verschiedenen Ausprägungsformen hinsichtlich der Drop-out-Gründe, Umgangsweisen mit dem Drop-out und Handlungsmöglichkeiten durch unterschiedliche Rahmenbedingungen rekonstruiert werden. In die Analysen wurden dabei nur Abbruchserfahrungen (Beendigung der Teilnahme vor dem regulären Ende) in Erwachsenenbildungsangeboten einbezogen, die den Kriterien der vom Deutschen Bildungsrat (1970) formulierten Definition von Weiterbildung genügen. Das Spektrum umfasste damit berufliche und allgemeine Weiterbildungsangebote: Von den Interviewteilnehmenden eingebrachte Abbruchserfahrungen in anderen Bildungssituationen (z. B. im Studium oder in der Ausbildung) wurden somit bspw. nur insofern berücksichtigt, wie sie für die Analyse und das Verständnis des Einzelfalls relevant wurden.

Neben der Einzelfallanalyse, die Strukturen und Muster aufzeigt, wurden über fallübergreifende, vergleichende Analysen in einem zweiten Schritt Typen anhand der deduktiv und induktiv erarbeiteten Kategorien entwickelt (vgl. Kluge 1999). Diese empirisch begründete Typologie ist eine mehrdimensionale Typologie (vgl. Kelle und Kluge 2010), dargestellt in einer Mehrfeldertafel (s. Abschn. 4.2) mit allen theoretisch möglichen Kombinationen der Merkmalsausprägungen. Die beiden zentralen Vergleichsdimensionen (die am Drop-out beteiligten Akteurinnen und Akteure sowie die Kontexte) ermöglichen in ihrer Kombination eine Beschreibung unterschiedlicher Arten von Nicht-Passung. Diese Vergleichsdimensionen wurden zunächst induktiv erarbeitet und im späteren Analyseprozess in einem ergänzenden deduktiven Vorgehen mit theoretischen Modellen (neben vielen anderen Kategorien, ua. der sozialen und fachlichen Integration, adaptiert nach Tinto) in Bezug gesetzt und differenziert. Beim Vergleich mit dem Forschungsstand wurde klar, dass auch Stamm et al. (2012) in ihrer Typenbildung zu Schulabbrüchen ähnliche Vergleichskategorien (Akteurinnen und Akteure, Kontexte) induktiv-deduktiv gebildet haben. Der Unterschied besteht jedoch in den Typen selbst, die bei uns Nicht-Passung fokussieren, bei Stamm Schulabbrechende als Personentypologie. Es handelt sich hierbei somit nicht um eine Personentypologie, sondern eine Typologie der NichtPassungen bei Drop-out.

Im Lauf der Fallanalysen und -vergleiche kristallisierte sich Passung/Nicht-Passung als Kernkategorie und als zentrales Phänomen heraus. Zur kontextuellen Einbettung der Typen und visuellen Verdeutlichung des prozesshaften Charakters wurde vor diesem Hintergrund das „Modell der situativen (Nicht-)Passung“ entwickelt, das in diesem Beitrag vorgestellt wird (siehe Abschn. 4.3). 


\section{Kernergebnisse: Drop-out im Lichte von Passungsfragen}

Während der Auswertung der Interviews mit den Abbrecherinnen und Abbrechern rückte vor allem das Phänomen der Passung in den Vordergrund - anfangs noch als vager Arbeitsbegriff für das Explizieren erster Erkenntnisse, zunehmend empirisch fundiert und im fortschreitenden Projektschritt der Analyse auch theoretisch elaboriert. Auf diese Weise erfolgte eine wechselseitige Inspiration von Empirie und Theorie, welche erst durch die Empirie relevant wurde. Dadurch wurden empirische Ergebnisse in eine theoretische Diskussion überführt und diese konnte wiederum als produktives Irritationsmoment an das empirische Material herangetragen werden. Diese Auseinandersetzung ist Teil der Projektergebnisse und soll folgend insoweit dargelegt werden, wie es der Nachvollziehbarkeit der Ergebnisse dienlich ist. Zunächst wird das im Verlauf des Forschungsprojekts erarbeite Verständnis des Passungsbegriffes dargestellt (vgl. Abschn. 4.1), welches sowohl der erarbeiteten „Туpologie der Nicht-Passung von Drop-out in der Weiterbildung“ (vgl. Abschn. 4.2), als auch dem daraus weiterentwickelten „Modell der situativen (Nicht-)Passung“ (vgl. Abschn. 4.3) zugrunde liegt.

\subsection{Theoretische Fundierung des Passungsbegriffs}

In der Erziehungswissenschaft wird der Begriff „Passung“ überwiegend sozialisationstheoretisch gefasst und auf Spannungsverhältnisse zwischen Habitus und Feld zurückgeführt (vgl. u.a. Kramer 2017; Meister und Sotzek 2017; Kramer und Helsper 2010). Der Passungsbegriff wird dabei nicht statisch gesehen, stattdessen wird ihm der Aspekt eines dynamischen Aushandlungsmomentes zugeschrieben (vgl. Meister und Sotzek 2017, S. 4). Dies bedeutet, dass sich objektiv-strukturelle als auch subjektiv erschlossene Bezugsrahmen und somit das „objektive Passungsverständnis“ sowie die „subjektive Passungswahrnehmung“, wie es Düggeli und Neuenschwander (2015) für Entscheidungsprozesse in der Berufsbiographie formulieren, über die Zeit hinweg verändern können. Dieses Begriffspaar bringt zudem eine weitere Perspektive ein: Passungen oder Nicht-Passungen hängen von objektiven wie subjektiven Faktoren gleichermaßen ab. Dabei bestimmt die individuelle Passungseinschätzung des Individuums immer auch den Blick auf die objektive Passung (vgl. ebd.).

Wendet man sich nun dem Begriff in der Erwachsenen- und Weiterbildung zu, ist er vor allem für die Mesoebene (Programmplanungs- und Adressatenforschung) und Mikroebene (Lehr-/Lernforschung, auch Kursforschung) elaboriert. In der LehrLernforschung geht es um Fragen der Passung von Raum und Zeit (siehe beispielsweise Studien von Kraus 2015; Herrle und Nolda 2010; Seitter 2017) und um Aspekte der Passung der Lehrstile von Dozierenden und den Aneignungsweisen/ Lernstilen der Teilnehmenden (Schrader 1994), also um eine Passung von Lernenden und Lernangeboten.

Theoretisch rückgebunden wird der Begriff dabei vor allem an Tietgens (1981), der in Anlehnung an Mitscherlich (1963) - bezogen auf das Programmplanungshandeln als didaktische Aufgabe von Erwachsenenbildnerinnen und Erwachsenenbildnern - von anthropologischen Suchbewegungen spricht (vgl. Tietgens 1982): Sowohl Planende als auch potenzielle Teilnehmende befinden sich in einer Suchbewegung, 
sodass sich Erwachsenen- und Weiterbildungseinrichtungen ,in ihrer meso- und mikrodidaktischen Planung an antizipierten und noch zu weckenden Bedürfnissen und Interessen der Adressat*innen [orientieren], während selbige ihrerseits Möglichkeiten suchen, ihre manifesten und latenten Lernbedürfnisse zu befriedigen“" (von Hippel et al. 2019, S. 86). Nur bei einer Konkordanz der verschiedenen Erwartungen der Planenden und Adressaten bzw. Adressatinnen kommt eine Weiterbildungsteilnahme zustande (vgl. Tietgens 1982).

Für eine weitere Präzisierung kann auch Schäffter (1992) herangezogen werden, der auf eine „Spannung zwischen Personen und Sache oder zwischen Situation und institutioneller Struktur“ (Schäffter 1992, S. 176f.) abhebt, in die immer auch die „Abstimmung von objektiven Lernanforderungen und subjektiven Lernvoraussetzungen“ (ebd., S. 177) inkludiert ist, sodass er in dem Begriff der Passung den „Ausdruck eines Strukturkonflikts“ (ebd.) sieht: „Es geht um ein Spannungsfeld, das sich auftut beim Aufeinandertreffen von kaum miteinander vermittelbaren Daseinsauslegungen, differenten Strukturen und Mentalitäten, heterogenen Stoffstrukturen und Wissensbeständen sowie verschiedenen Erwartungshaltungen und Motivbündeln“ (ebd., S. 179).

Diese Definition nach Schäffter kommt dem Passungsverständnis nahe, welches auch in der Drop-out-Literatur genutzt wird. Der Passungsbegriff spielt in dieser insofern eine Rolle, als dass ein „Dropout [...] zunächst verstanden werden [kann] als eine fehlende Passung von Wünschen, Bedürfnissen und Interessen der Lernenden einerseits sowie der Gestaltung der Rahmenbedingungen des Angebots andererseits“ (Schmidt 2011, S. 206f.). Auch hier geht es also um eine Passung zwischen den Akteurinnen und Akteuren der Programmplanung und den Adressatinnen und Adressaten bzw. Teilnehmenden, bzw. um ein Bemühen um eine Passung zwischen Bildungsangebot und Bildungsnachfrage (vgl. Brödel 1996, S. 26). Dabei wird angemerkt, dass die Passung sich im Laufe einer Bildungsveranstaltung durchaus verändern kann, da sich Bildungsinteressen von Teilnehmenden über einen Kurs hinweg ggf. erst entwickeln oder offenbaren (vgl. Schmidt 2011, S. 207). In Bezug auf Drop-out-Entscheidungen werden durchgängig Passungseinschätzungen seitens der Teilnehmenden fokussiert; Passungseinschätzungen seitens der Bildungseinrichtungen, die ggf. zu einem Drop-out seitens dieser führen können, wurden bisher jedoch nicht für die Erklärung von Drop-outs betrachtet.

Aus diesen theoretischen Bezügen resultiert ein Verständnis von Passung, das nicht von einem objektiv beobachtbaren Phänomen ausgeht, sondern von Passung als relationalem Konstrukt (vgl. Emirbayer 1997). Wird diese Passung von mindestens einer Seite (Individuum oder Institution) als nicht hinreichend bewertet, und wird keine Möglichkeit zur (Wieder-)Herstellung einer hinreichenden Passung oder zur Toleranz der Nicht-Passung gesehen, so kommt es zum Drop-out. Der Begriff der Passung als Ziel anthropologischer Suchbewegungen impliziert ein Spannungsverhältnis zwischen einem Individuum und dem (Weiterbildungs-)Umfeld, in dem es sich temporär bewegt. Aspekte, die zwischen diesen beiden Kontexten und den ihnen inhärenten Akteursgruppen nicht passend sein können, sind u. a. beidseitige Erwartungen, Motive, Wünsche, Bedürfnisse, Bedarfe, Inhalte, Interessen, Anforderungen und Voraussetzungen für Teilnahme und Abschluss einer Weiterbildung, Lehr-/Lernkulturen, vermittelte Inhalte und weitere Rahmenbedingungen des An- 
gebotes (wie Zeiten, Kosten, Lehr-/Lernsetting usw.). Aufgrund all dieser Aspekte und der Bewertung dieser durch die verschiedenen Akteursgruppen bilden sich letztlich Passungseinschätzungen seitens der Individuen als auch der Institutionen aus. Diese können einen objektiven (z. B. ausformulierte Kriterien, wie Zugangsvoraussetzungen) als auch subjektiven Charakter (z. B. nicht ausformulierte Kriterien, wie Nutzenerwartungen) haben. Sie sind dabei mitnichten starr, sondern unterliegen dynamischen, situativen Aushandlungsprozessen vor der Folie der eigenen Sozialisation, eigenen vorgängigen Erfahrungen, Bewertungsschemata usw. und gehen sowohl mit kognitiven/rationalen als auch emotionalen Anteilen einher.

\subsection{Typologie zur Nicht-Passung bei Drop-out in der Weiterbildung}

Folgende Typen ergaben sich aus der empirischen Analyse der Interviews mit den Abbrechenden und geben Hinweise zur Beantwortung der übergeordneten Forschungsfrage des Projekts „Analyse von Drop-out in der Weiterbildung“ (siehe Abschn. 3), in welchen Konstellationen es zu Drop-outs kommt und welche Gründe als ausschlaggebend für Drop-out angesehen werden. Die Typen bilden die Varianz von Konstellationen der Nicht-Passung ab, die als ein signifikanter Grund für Dropouts in Weiterbildungen und als Kernkategorie herausgearbeitet wurden.

Passungsbedingte Drop-outs werden auf verschiedenen Ebenen evoziert - auf der institutionellen genauso wie auf der individuellen. Das ist darin begründet, dass auch Akteurinnen und Akteure, die an einem Drop-out beteiligt sind, sowohl Individuen in ihrer Lebenswelt als auch Institutionen sein können. Aus der Verschränkung dieser Aspekte in einer Kreuztabelle entstand so eine „Typologie von Nicht-Passungen von Drop-out in der Weiterbildung“" (vgl. Abb. 1), welche sieben Ausprägungen von Nicht-Passungen umfasst.

Der Typ Intraindividuelle Nicht-Passung deutet auf eine Nicht-Passung innerhalb eines Subjektes in Bezug auf überdauernde und temporäre/situativ erforderliche Formen im Umgang mit der Welt und sich selbst. Ein Drop-out kommt zustande, wenn überdauernde Merkmale auch in der Weiterbildungssituation auftreten und dazu führen, dass eine Teilnahme nicht mehr möglich ist. Diese stabilen Muster im Umgang mit sich und der Welt stehen somit einer erfolgreichen Teilnahme entgegen und ein Drop-out stellt eine Möglichkeit dar, eine Nicht-Passung aufzulösen und (wieder) eine Passung innerhalb des Subjekts herzustellen, schließlich werden diese (intraindividuell ausgetragenen) Konfliktsituationen aufgelöst. Empirisch zeigt sich dies z.B. im Falle eines Besuchs einer Weiterbildung zur Kontaktimprovisation, in welcher der Teilnehmer durch seine Aversion gegen Strukturen (die sich im biographischen Verlauf in mehreren Lebensbereichen manifestiert) auch in dieser Weiterbildung ein Unwohlsein entwickelt und die Weiterbildung schließlich vorzeitig beendet.

Der Typ Lebenskontextuelle Nicht-Passung verweist auf sich verändernde Lebensumstände, die eine Weiterbildung irrelevant werden lassen. Empirisch findet sich dieser Typ in einem Fall der Weiterbildung als Fiction Producer wieder, die dem Teilnehmer beim Wechsel des angestammten Berufsfeldes (Journalismus) in das anvisierte Berufsfeld (Filmproduktion) von Nutzen sein soll. Während der Weiterbildung lernt er das anvisierte Feld weitergehend kennen und erkennt, dass er in 


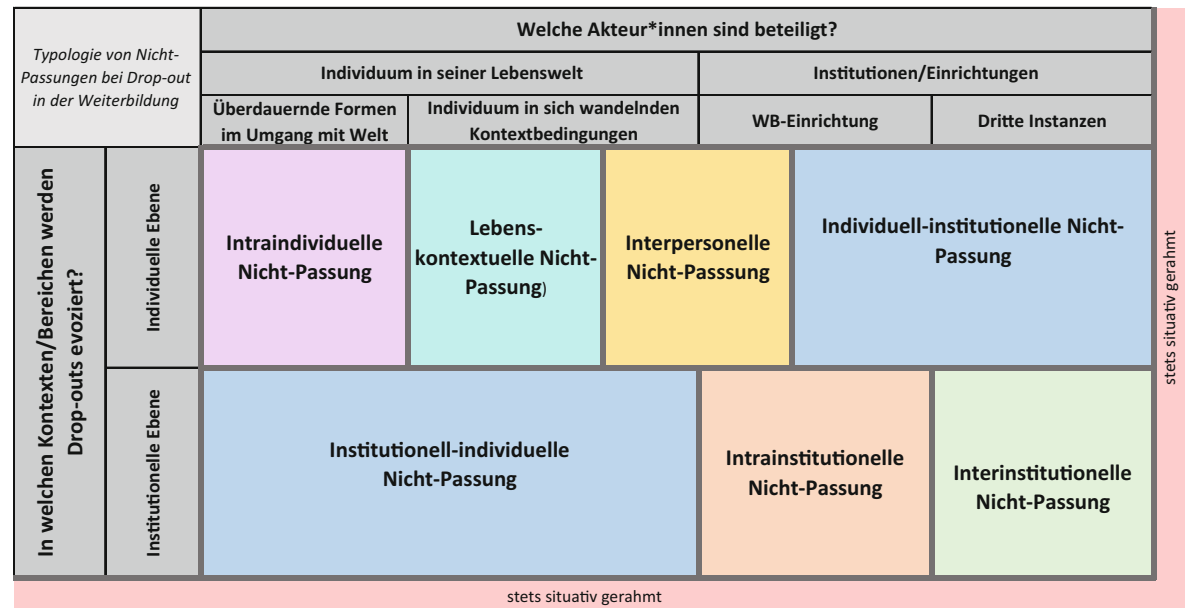

Abb. 1 Typologie von Nicht-Passungen bei Drop-out in der Weiterbildung

diesem Feld aus verschiedensten Gründen nicht arbeiten möchte, sodass die Weiterbildung ihre Relevanz verliert und er die Weiterbildung vorzeitig beendet.

Fokussieren die beiden zuvor genannten Typen noch ausschließlich das Individuum und seine private sowie berufliche Lebenswelt, rückt im Typ Interpersonelle Nicht-Passung nun auch der Weiterbildungsbereich in den Blick. In einer Weiterbildungssituation treffen mindestens zwei Personensysteme innerhalb eines Weiterbildungs- und weiterer Kontexte aufeinander, wodurch es aufgrund von unterschiedlichen Erwartungen o. ä. zu interpersonellen Nicht-Passungen kommen kann, die Ausdruck in heißen und kalten Konflikten finden können. Nicht gemeint sind hier Konflikte mit institutionell begriffenen Personen wie Dozentinnen und Dozenten, sondern Konflikte zwischen z. B. Individuum und anderen Teilnehmenden oder zwischen Individuum und Partnerin oder Partner. Zur Illustration kann hier ein Fall aus der Empirie herangezogen werden, in dem die Teilnehmerin eines EDV-Kurses zur Zeit einer Ehekrise entgegen den Wünschen und Erwartungen ihres Ehepartners agiert, der seine Ehepartnerin mit einem Teilzeitjob und der Kinderbetreuung als ausgelastet einschätzt. Sie bricht die Weiterbildung u.a. ab, da die Teilnahme mit Konflikten in ihrem privaten Umfeld einhergeht und sie nicht die Unterstützung von ihrem Partner und der erweiterten Familie erhält, die sie sich wünscht, um mit der Mehrbelastung der Weiterbildung umgehen zu können.

Der Typ Individuell-institutionelle Nicht-Passung verweist auf eine Diskrepanz zwischen Individuum und Institutionen, wie der Weiterbildungsinstitution an sich, oder weiteren Institutionen, wie einem Amt o.ä.: Vom Individuum ausgehende Erwartungen, formulierte Anforderungen an Institutionen, Passungseinschätzungen und Interesselagen passen von Beginn an nicht oder über die Weiterbildungsaktivität hinweg nicht mehr. Unzufriedenheiten des Individuums gegenüber der Institution werden ggf. thematisiert, können allerdings nicht aufgelöst werden, sodass es zu einem Drop-out seitens des Individuums kommt. Empirisch zeigt sich dies im Fall eines Weiterbildungsbesuchs zur Yogalehrerin, der unter bestimmten Prämissen 
zwischen Teilnehmerin und Weiterbildungsanbieterin ausgehandelt wurde (u. a. zeitliche Flexibilisierung der Ausbildung und individueller Preisnachlass von 50\% der Teilnahmegebühren), in dem diese Vereinbarung seitens der Weiterbildungsanbieterin jedoch nicht eingehalten wurde. Der Diskrepanz zwischen Erwartungen und der Weiterbildungsrealität begegnet die Teilnehmerin mit einem vorzeitigen Abbruch.

Diese Diskrepanz greift auch der Typ Institutionell-individuelle Nicht-Passung auf, allerdings gehen die Unzufriedenheit und der Drop-out von der Institution (z. B. Arbeitsamt, Jobcenter, Weiterbildungseinrichtung) aus, wenn als objektiv angesehene Anforderungen als nicht gegeben eingeschätzt werden. Ein empirischer Fall, in dem sich dieser Typ widerspiegelt, ist der einer Teilnahme an einer Weiterbildung im Bereich Kunsttherapie. Als die Teilnehmerin innerhalb der Weiterbildung von einem kürzlich zurückliegenden, stationären Klinikaufenthalt berichtet, schließt die Weiterbildungseinrichtung sie kurzerhand wegen unzureichender emotionaler Stabilität aus. Die Passungseinschätzung der Weiterbildungseinrichtung wandelte sich also im Laufe der Zeit, sodass sie den Drop-out ihrerseits vollzieht.

Im Gegensatz zu den bisherigen Typen, die alle das Individuum berühren, führen die beiden letzten zu einem Drop-out, ohne dass das Individuum selbst daran beteiligt, jedoch davon betroffen ist. Der Typ Intrainstitutionelle Nicht-Passung zeigt auf, dass sich der Drop-out-Prozess auf einer Mesoebene abspielt und das Individuum davon auf einer Mikroebene betroffen wird, ohne dass es an diesen Prozessen teilhatte. Ein solcher Drop-out kann - rekonstruiert aus den Interviews mit Expertinnen und Experten - zum Beispiel stattfinden, wenn eine Institution in sich selbst NichtPassungen verzeichnet, z.B. zwischen der Einrichtung und für eine Veranstaltung beauftragte Lehrperson, die diesen Auftrag dort nicht mehr weiter wahrnimmt. So kann ein Kurs ggf. nicht weiter stattfinden, was auch einen Drop-out der Teilnehmenden nach sich ziehen würde.

Der Typ Interinstitutionelle Nicht-Passung verweist darauf, dass eine Nicht-Passung auch zwischen institutionellen Instanzen entstehen kann, so zum Beispiel zwischen einem die Weiterbildung finanzierenden Arbeitgeber und der Einrichtung/dem Angebot der Einrichtung oder zwischen der Arbeitsagentur als Mittelgeberin und der Weiterbildungseinrichtung. Empirisch zugespitzt zeigt sich dies auch im Fall einer Weiterbildungsteilnahme, die selbstgewählt im Rahmen eines Gefängnisaufenthalts stattfindet. Die Teilnehmerin besucht dort einen Microsoft-Office-Kurs, muss diesen jedoch abbrechen, da auch ein Kuraufenthalt in dieser Zeit veranschlagt wird. Sie verlässt daraufhin das Gefängnis und muss infolgedessen die Weiterbildung vorzeitig beenden, obwohl sie diese gern fortgesetzt hätte. Hier gab es Nicht-Passungen in den Absprachen bzw. Interessen des Gefängnisses und weiteren Institutionen, sodass ein Drop-out ohne das Zutun der Abbrecherin vollzogen wird.

\subsection{Das Modell der situativen (Nicht-)Passung zur Betrachtung von Drop-out}

Aufbauend auf die vorangestellte ,Typologie zur Nicht-Passung von Drop-out in der Weiterbildung" (siehe Abschn. 4.2), die eine Varianz von Konstellationen von Nicht-Passungen als Gründe für Drop-outs in der Weiterbildung in den Vordergrund stellt, rückt in einer prozesshaften Betrachtung - für welche Elemente des Kodierparadigmas herangezogen wurden, mit dessen Hilfe sich (Nicht-)Passung zuvor als 
zentrale Kategorie rekonstruieren ließ - die Frage nach der situativen Passungseinschätzung in den Mittelpunkt. So lassen sich Drop-outs aufgrund von Nicht-Passung noch besser in einer chronologischen Verkettung von Bedingungen, Einschätzungen und Handlungen betrachten und weniger als singuläres, plötzliches Ereignis. Dies ist im „Modell der situativen (Nicht-)Passung“ zur Betrachtung von Drop-out visuell dargestellt (vgl. Abb. 2) und wird im Folgenden näher erläutert.

Mit Blick auf den linken Teil des Modells wird deutlich: Passungseinschätzungen gibt es bereits vor Beginn der Teilnahme an und somit ab dem Zeitpunkt der Entscheidungsphase für oder gegen eine Weiterbildungsteilnahme. Dabei kann eine Entscheidung nicht nur vom Individuum ausgehen, welche sich aufgrund individueller (Dispositionen, Erwartungen, Wünsche, Teilnahmemotive, Interessen, Lebenswelt, Bildungserfahrungen, Lernkultur, Ressourcen, Weiterbildungseinstellungen etc.) und institutioneller Bedingungen (bildungspolitischer Rahmen, Angebot, Lerninhalte, Ausstattung, Personal, Teilnehmendengruppe, Anforderungen, Voraussetzungen, Lehrkultur, Kosten etc.) ausformt, sondern auch von Institutionen wie der Agentur für Arbeit, dem Jobcenter, der Weiterbildungseinrichtung, dem Arbeitgeber usw. (wenn z.B. Kriterien für eine Förderung der Weiterbildung nicht erfüllt sind, potenzielle Teilnehmende die Zugangskriterien zu einer Weiterbildung nicht erbringen oder der Arbeitgeber die Weiterbildung anordnet). Bei der Entscheidung für eine Weiterbildungsteilnahme ist davon auszugehen, dass mehrere Beteiligte eine positive Passungseinschätzung entwickelt haben. Dabei sollten Bedingungen für Passungseinschätzungen bzw. das Verhalten in diesen Entscheidungssituationen nicht nur in die Akteurinnen und Akteure (Teilnehmende, Mitarbeitende der Weiterbildungseinrichtung, Mitarbeitende der Agentur für Arbeit usw.) verlagert werden, denn diese Bedingungen sind stets abhängig von der soziostrukturellen Rahmung und damit einhergehenden Strukturen, Ressourcen, Möglichkeiten, Grenzen usw. Dies wirkt nicht nur in dieser Phase, sondern auch in den weiteren Phasen der Weiterbildungsteilnahme und des Abbruchs.

Mit Blick auf den mittleren Teil des Modells ergibt sich, dass sich die situative Passung aus dem Zusammenspiel der individuellen sowie institutionellen Passungseinschätzung ergibt, die sowohl explizit als auch vorbewusst wahrgenommen werden kann - von den Teilnehmenden selbst als auch von Akteurinnen und Akteuren verschiedener Institutionen (siehe Abschn. 4.2). Dabei ist ein Passungsempfinden situativ und über den Prozess der Weiterbildungsteilnahme (und darüber hinaus) veränderbar.

Umso ausgeprägter die Passungseinschätzungen insgesamt, desto höher ist auch die Wahrscheinlichkeit für einen Verbleib in einem Kurs (und vice versa). Allerdings wiegen sich die einzelnen Bereiche der Passungseinschätzungen nicht unbedingt gegeneinander auf, sodass es bei positiven Passungseinschätzungen in den meisten Bereichen und gleichzeitiger Nicht-Passung in einem der Bereiche durchaus zu einem Drop-out kommen kann.

Im rechten Teil des Modells wird noch einmal der reversible und nicht-lineare Prozesscharakter von (drohenden) Drop-outs aufgrund von Nicht-Passungen deutlich. Situationsüberdauernde positive Passungseinschätzungen von individueller und institutioneller Seite führen in der Konsequenz zu einem Verbleib in der Weiterbildung. Negative Passungseinschätzungen seitens der Individuen und Institutionen 


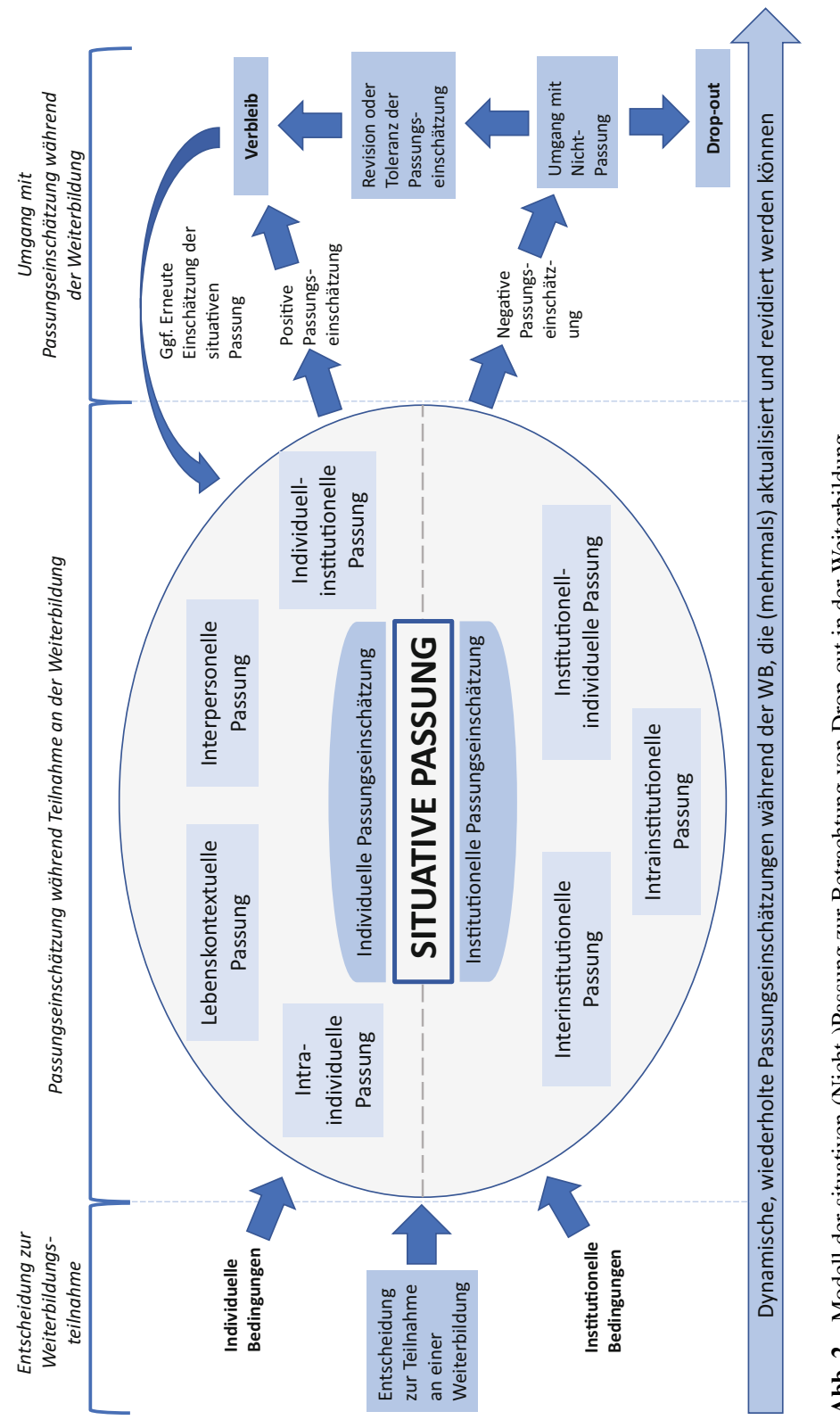


lassen einen Verbleib ungewisser werden und erfordern einen Umgang mit dieser Einschätzung zur Nicht-Passung. Dies kann zu abweichenden Ergebnissen führen: Zum einen kann es (a) zur Toleranz der negativen Passungseinschätzung kommen. Möglich ist auch, dass es (b) zu einer Revision der Einschätzung führt (z.B. durch Beratungen, Reflexionen, selbst oder von außen herbeigeführte Veränderungen der Situation, oder auch durch Umdeutungen, Veränderung von Kriterien, konfliktlösende Gespräche) und dadurch zu einer Entscheidung des Verbleibs in der Weiterbildung. Ggf. kommt es dann während der Weiterbildungsteilnahme zu einer erneuten Einschätzung der situativen Passung. Zum anderen kann es (c) aufgrund negativer Passungseinschätzungen zu einem unmittelbaren Drop-out durch die Teilnehmenden oder die Einrichtung kommen, der eine weitere Kursteilnahme oder einen späteren Wiedereinstieg in der Regel ausschließt. Ggf. wird seitens des Individuums durch einen Drop-out an dieser Stelle wieder eine Passung (in Bezug auf eigene Überzeugungen o. ä.) hergestellt, auch wenn es dazu führen kann, dass Nicht-Passungen (z.B. zwischen Person und Qualifikationsanforderungen für einen Job) im weiteren Lebensverlauf bestehen bleiben.

Wird das Modell von links nach rechts gelesen, wird ersichtlich, dass Passungseinschätzungen prospektiv, situativ als auch retrospektiv eingenommen und in der spezifischen Situation der (vorzeitig endenden) Weiterbildungsteilnahme von individuellen, institutionellen und soziostrukturellen Aspekten berührt werden. Die Erfahrungen, die eine Person bis zum aktuellen Zeitpunkt gesammelt hat, wirken sich in einer nächsten Weiterbildung wiederum auf die im Modell abgebildeten Phasen aus, schließlich haben sie auch Einfluss auf künftiges Weiterbildungsverhalten.

\subsection{Schlussfolgerungen aus diesen Betrachtungen}

Schlussfolgerungen können in mindestens zweierlei Hinsicht gezogen werden: Einerseits ermöglichen die ,Typologie zur Nicht-Passung von Drop-out in der Weiterbildung“ und das „Modell der situativen (Nicht-)Passung“ eine Verdichtung von Annahmen rund um Drop-out in der Weiterbildung, auch wenn nach wie vor Fragen offenbleiben - bzw. durch diese Ergebnisse erst aufgeworfen werden. Andererseits ermöglichen die Ergebnisse auch Rückschlüsse und Spezifizierungen hinsichtlich des herangezogenen Begriffs der Passung.

In den Ergebnissen dieser Studie spiegelt sich das unter Abschn. 4.1 ausgeführte Verständnis von Passung umfänglich wider, das dynamische Aushandeln und der Blick auf Passungseinschätzungen werden sichtbar. Eine Ausdifferenzierung erfährt der Begriff hinsichtlich der Erkenntnisse, dass auch Passungsverhältnisse zwischen und innerhalb von Institutionen (ohne Beteiligung der Teilnehmenden) einen Dropout evozieren können und dass nicht nur Spannungen zwischen Teilnehmenden und Einrichtungen bzw. zwischen den Institutionen, sondern auch Spannungen innerhalb des Individuums, zu negativen Passungseinschätzungen und Drop-outs führen können. Keine gänzlich neue Erkenntnis, aber eine, die in diesem Kontext emergierte und herausgestellt werden soll, ist der Umstand, dass Drop-outs oft in einem problematisierenden Kontext diskutiert werden, sich in der Empirie aber nicht nur als Erleiden, sondern auch als Selbstermächtigung und positiv Erlebtes zeigen. 
Diese Betrachtungsweisen schließen an bisherige Drop-out-Forschungen an und weisen in Zugang, Ergebnissen und Schlussfolgerungen zahlreiche Parallelen, aber auch abweichende Perspektiven auf: Während Stamm et al. (2012) z.B. ähnlich in der Tabellierung zur Typenbildung vorgehen, bezeichnet sie mit Drop-outs Personen(gruppen). In dem hier beschriebenen Forschungszusammenhang richtet sich die Betrachtung hingegen allgemein auf den Prozess und in der Typenbildung auf Abbruchsgründe statt auf Personentypen. Prozesshaftigkeit, der in dem ,Modell der situativen (Nicht-)Passung“ Rechnung getragen wird, spielt auch in den Modellen von Tinto und Boshier eine Rolle: Beide reduzieren Drop-outs nicht auf monokausale Annahmen, sondern beziehen subjekt- als auch gesellschaftsbezogene Kategorien zur differenzierten Betrachtung mit ein, die verdeutlichen, dass die Abbrecherinnen und Abbrecher genauso wie die Umwelt - bzw. eine Melange aus diesen - ursächlich für den Ausgang von Weiterbildungssituationen sein können. So rücken sowohl Tintos Begrifflichkeiten, wie die soziale und akademische Integration (vgl. Tinto 1975, 1993), als auch Boshiers Begriffe der Kongruenz und Inkongruenz (vgl. Boshier 1973) in die Nähe des skizzierten Passungsbegriffs. Gemeinsam ist allen drei Modellen die Frage nach der Beziehung von den Lernenden und weiteren Faktoren in Weiterbildungssituationen. Mit dem „Modell der situativen (Nicht-)Passung“ liegt nun ein ergänzendes Modell zur Erklärung von Nicht-mehr-Teilnahmen vor, das durch den Bezug auf den Passungsbegriff und aktuelle empirische Ergebnisse dazu beiträgt, die Betrachtung von Weiterbildungsabbrüchen auf theoretisch und empirisch noch breiteres Fundament zu stellen. Durch die Kombination von Kategorien in der Typenbildung werden Konstellationen von Passungseinschätzungen systematisch abbildbar und es ist so auch möglich, andere Akteurinnen und Akteure neben dem lernenden Individuum in die Betrachtung einzubeziehen (die letztgenannte ist eine Betrachtungsweise, die es in der Schulforschung zu Drop-out gibt, bislang aber in der erwachsenenpädagogischen Drop-out-Literatur kaum vorkam).

Auch wenn die ,Typologie der Nicht-Passung von Drop-out in der Weiterbildung“ und das „Modell der situativen (Nicht-)Passung“ weitere Erklärungsansätze für Drop-out in der Weiterbildung unterbreiten, unterliegen sie Limitationen: Der ersten Limitation, dass eine Typologie keine phasenbasierte Prozesshaftigkeit abbilden kann, konnte mit der Weiterentwicklung zu einem „Modell der situativen Passung" begegnet werden. Es kann jedoch in beiden Darstellungsweisen nur unzureichend abgebildet werden, wie interdependent die Faktoren sind, die auf Drop-outs wirken, da die einzelnen Fälle (welche durchaus dezidierte Rückschlüsse auf multifaktorielle Überlagerungen zulassen) im Rahmen der Typen- und Modellbildung nach dominanten Merkmalen verortet wurden, um sie im Rahmen einer Bündelung handhabbarer und vermittelbar zu machen. Zweitens beziehen sich die gebildeten Typen auf Gründe für Drop-outs und nicht auf personengruppenbezogene Typen, wodurch kein direkter Anschluss an die Personengruppen möglich ist, die in der ersten Projektphase mit Hilfe der NEPS-Daten herausgearbeitet wurden (vgl. Hoffmann et al. 2020). Drittens bestehen Limitationen in der Erklärungskraft der beiden Ergebnisformate aufgrund der dem Projekt zugrundeliegenden Drop-out-Definition (vgl. ebd.), da Abbrechende ihr Teilnahmeverhalten z. T. als Drop-out beurteilen, diese Fälle jedoch nicht weiter berücksichtigt wurden, da sie nicht dem im Projekt (theoretisch und empirisch durch die Interviews mit den Expertinnen und Experten) 
erarbeiteten Drop-out-Begriff entsprachen. Eine sukzessive Erweiterung des Begriffs könnte weitere Ausdifferenzierungen und Verdichtungen nach sich ziehen.

\section{Diskussion und Ausblick}

Anknüpfend an tradierte Diskurse und theoretische Modelle zur Frage von Teilnahme und Nicht-Teilnahme an Weiterbildung wird mit der vorliegenden Untersuchung mit dem Fokus auf die Nicht-mehr-Teilnahme ein Aspekt hinzugefügt, der in der erwachsenenpädagogischen Theoriebildung bislang kaum eine Rolle gespielt hat. Die Konzepte von Tinto und Boshier aus den 1970er Jahren bieten zwar einen ersten theoretischen Bezugspunkt, erweisen sich jedoch hinsichtlich der Vielfalt und Spezifität von Weiterbildungsabbrüchen als nicht hinreichend erklärungsmächtig. Mit dem Konzept der Passung bzw. Nicht-Passung schlagen wir eine Erweiterung der genannten theoretischen Zugänge vor, die - so zumindest das Fazit unserer empirischen Analysen - einen wesentlichen Beitrag zur Differenzierung und zum Verständnis von Weiterbildungsabbrüchen leisten kann. Dabei ist Passung ein stets subjektiver, fragiler, situationsbezogener Zustand, der sowohl durch die Interaktion der Lernenden untereinander, mit den Lehrenden und mit der Organisation (re-)produziert oder reduziert werden kann, als auch im Rahmen der Koordination unterschiedlicher institutioneller Akteurinnen und Akteure und Lebensbereiche.

Das in einem an die Grounded-Theory angelehnten Forschungsprozess als Kernkategorie herausgearbeitete Konzept der Passung ist gleichzeitig Grundlage für die hier vorgestellte Typologie von Weiterbildungsabbrüchen. Dabei kann das Passungskonzept durchaus als theoretisches Konstrukt verstanden werden, das unmittelbar anschließt an vorangegangene theoretische Diskurse und Modelle (z. B. Kramer 2017; Meister und Sotzek 2017; Düggeli und Neuenschwander 2015). Die Typologie als „Zwischenschritt der Theoriebildung“ (Kluge 1999, S. 51), der den Weg bereitet von empirischen Analysen hin zur Theorieentwicklung, erweitert den binären Blick der Teilnahmeforschung auf Teilnahme und Nicht-Teilnahme um die Perspektive der Nicht-mehr-Teilnahme.

Damit treten Teilnahme und Nicht-Teilnahme als Pole eines Kontinuums in Erscheinung, mit vielfältigen Zwischenformen. So bleibt zum Beispiel die Frage vorläufig offen, inwieweit das Konzept der Passung auch zur Analyse der eigentlichen Weiterbildungsteilnahme vorgelagerter Abbrüche tragfähig ist oder welche Formen von Teilnahmeunterbrechungen in Weiterbildungskontexten relevant werden. Da mit dem Sample der beschriebenen Studie Abbrüche fokussiert werden und daher auch nur Aussagen zu Passungseinschätzungen bei Abbrüchen vorgenommen werden können, wäre es spannend, in folgenden Studien Verläufe in der (fortgesetzten) Teilnahme unter Passungsfragen zu betrachten.

Im Sinne eines forschungsbezogenen Ausblicks lassen sich weitere Anschlussmöglichkeiten formulieren. In dem vorliegenden Projekt wurden Drop-outs während der Teilnahme untersucht, ein Forschungsdesiderat ist auch die Untersuchung von sehr frühen Drop-outs, also vor Beginn der Teilnahme. Interessant wäre es darüber hinaus, längsschnittlich Prozesse der Revision negativer Passungseinschätzung und den Verbleib in der Weiterbildung zu untersuchen, um Drop-out-Verhalten in seiner 
Spezifik tiefergehend zu verstehen. Darunter fallen auch die Fragen, ab wann eine Passung von verschiedenen Akteurinnen und Akteuren als zu minimal eingeschätzt wird und wovon das wiederum abhängt. Wieviel Nicht-Passung ist u. U. auch lernförderlich, insbesondere auf der mikrodidaktischen Ebene? Da Passung ein starkes Thema in der Lehr-Lernforschung und Kursforschung ist, könnten hier Beobachtungen zusammengebracht werden. Der Umgang mit Passungsphänomenen durch die professionell Handelnden auf Meso- und Mikroebene könnte auch aus professionstheoretischer Perspektive analysiert werden als der Umgang mit professionellen Antinomien, so spiegeln sich in einigen Typen der Nicht-Passung auch professionelle Antinomien auf Seiten der professionell Handelnden (z. B. Begründungsantinomie und Sachantinomie (vgl. Helsper 2002; von Hippel 2011)). Zusammenfassend liegt das Thema Drop-out und Passung an der Schnittstelle verschiedener Forschungsbereiche: der Adressaten- und Teilnehmendenforschung (deren Perspektive in diesem Beitrag eingenommen wurde), der Programmplanungsforschung sowie der LehrLernforschung und auch der Beratungsforschung.

Für einen praxisbezogenen Ausblick lassen sich aus dem „Modell der situativen (Nicht-)Passung“" und der Typenbildung Anschlüsse für die Weiterbildungspraxis herstellen. Typenbildungen haben das Potenzial, auf ihrer Grundlage handlungsrelevantes Wissen für die Praxis ableiten zu können (vgl. Schmidt-Hertha und Tippelt 2011, S. 23), hier z. B. für den Umgang mit Drop-out in Weiterbildungsinstitutionen auf lehrender, planender und beratender Ebene, aber auch in anderen beteiligten Institutionen.

Förderung Das Projekt „Analyse von Drop-out in der Weiterbildung (Verbreitung, Einflussfaktoren, Auswirkungen): Entwicklung einer gegenstandsverankerten Theorieperspektive auf Drop-out" wurde von der Deutschen Forschungsgemeinschaft von 10/2017 bis 10/2019 unter folgenden Förderkennzeichen gefördert: SCHM 2391/6-1 und HI 1599/4-1.

Funding We acknowledge support by the Open Access Publication Fund of Humboldt-Universität zu Berlin and by the Projekt DEAL.

Open Access Dieser Artikel wird unter der Creative Commons Namensnennung 4.0 International Lizenz veröffentlicht, welche die Nutzung, Vervielfältigung, Bearbeitung, Verbreitung und Wiedergabe in jeglichem Medium und Format erlaubt, sofern Sie den/die ursprünglichen Autor(en) und die Quelle ordnungsgemäß nennen, einen Link zur Creative Commons Lizenz beifügen und angeben, ob Änderungen vorgenommen wurden.

Die in diesem Artikel enthaltenen Bilder und sonstiges Drittmaterial unterliegen ebenfalls der genannten Creative Commons Lizenz, sofern sich aus der Abbildungslegende nichts anderes ergibt. Sofern das betreffende Material nicht unter der genannten Creative Commons Lizenz steht und die betreffende Handlung nicht nach gesetzlichen Vorschriften erlaubt ist, ist für die oben aufgeführten Weiterverwendungen des Materials die Einwilligung des jeweiligen Rechteinhabers einzuholen.

Weitere Details zur Lizenz entnehmen Sie bitte der Lizenzinformation auf http://creativecommons.org/ licenses/by/4.0/deed.de.

\section{Literatur}

Bariso, E.U. (2008). Factors affecting participation in adult education: a case study of participation in Hackney and Waltham Forest, London. Studies in the Education of Adults, 40(1), 110-124. 
Boshier, R. (1973). Educational participation and dropout: a theoretical model. Adult Education, 23(4), 255-282.

Bourgeois, E. (1998). Introduction. In Verband Wiener Volksbildung (Hrsg.), Adult Learning and Social Participation (S. 331-335). Wien: Verband Wiener Volksbildung.

Brödel, R. (1996). Dropout. Kursabbruch in der Erwachsenenbildung. Unterrichtswissenschaft: Zeitschrift für Lernforschung, 24(1), 21-31.

d. Carmo Nicoletti, M. (2019). Revisiting the Tinto's theoretical dropout model. Higher Education Studies, 9(3), 52-64.

Deutscher Bildungsrat (1970). Strukturplan für das Bildungswesen. Stuttgart: Dt. Bildungsrat.

Düggeli, A., \& Neuenschwander, M.P. (2015). Entscheidungsprozesse und Passungswahrnehmung. In K. Häfeli, M.P. Neuenschwander \& S. Schumann (Hrsg.), Berufliche Passagen im Lebenslauf (S. 219-241). Wiesbaden: Springer.

Egloff, B. (2011). Kurs ohne Übergang? Teilnehmerinnen und Teilnehmer an Alphabetisierungskursen. In B. Egloff \& A. Grotlüschen (Hrsg.), Forschen im Feld der Alphabetisierung und Grundbildung. Ein Werkstattbuch (S. 175-190). Münster: Waxmann.

Egloff, B., Jochim, D., \& Schimpf, E. J. (2009). Zwischen Freiheitszugewinn, zugemuteter Emanzipation und Schaffung neuer Abhängigkeit. Kursbindung in der Alphabetisierung/ Grundbildung. REPORT - Zeitschrift für Weiterbildungsforschung, 32(4), 11-22.

Emirbayer, M. (1997). Manifesto for a relational sociology. American Journal of Sociology, 103(2), 281-317.

Flick, U. (2007). Qualitative Sozialforschung. Eine Einführung (8. Aufl.). Reinbek: Rowohlt.

Garrison, D. R. (1987). Researching dropout in distance education. Distance Education, 8(1), 95-101.

Gieseke, W. (2016). Lebenslanges Lernen und Emotionen. Wirkungen von Emotionen auf Bildungsprozesse aus beziehungstheoretischer Perspektive. Bielefeld: wbv.

Grotlüschen, A. (2010). Erneuerung der Interessetheorie. Die Genese von Interesse an Erwachsenen- und Weiterbildung. Wiesbaden: Springer VS.

Gruber, C. (1985). Determinanten des Kursabbruchs in der Erwachsenenbildung. Linz: Johannes Kepler Univ.

Helsper, W. (2002). Pädagogisches Handeln in den Antinomien der Moderne. In H.-H. Krüger \& W. Helsper (Hrsg.), Einführung in Grundbegriffe und Grundfragen der Erziehungswissenschaft (5. Aufl. S. 15-34). Opladen: Leske + Budrich.

Herrle, M., \& Nolda, S. (2010). Die Zeit des (Nicht-)Anfangens. Zum Prozessieren von Erreichbarkeit und Vermittlungsbereitschaft in der Etablierungsphase pädagogischer Interaktion. Zeitschrift für Pädagogik, 3, 340-354.

von Hippel, A. (2011). Programmplanungshandeln im Spannungsfeld heterogener Erwartungen. Ein Ansatz zur Differenzierung von Widerspruchskonstellationen und professionellen Antinomien. REPORT - Zeitschrift für Weiterbildungsforschung, 1, 45-57.

von Hippel, A., Kulmus, C., \& Stimm, M. (2019). Didaktik der Erwachsenen- und Weiterbildung. Paderborn: F. Schöningh.

Hoffmann, S., Thalhammer, V., von Hippel, A., \& Schmidt-Hertha, B. (2020). Drop-out in der Weiterbildung - eine Verschränkung von Perspektiven zur (Re-)Konstruktion des Phänomens Drop-out. Zeitschrift für Weiterbildungsforschung, 43(1), 31-46.

Jacot, A., Frenay, M., \& Cazan, A.-M. (2010). Dropout of adult learners returning to university. Interactions of motivational and environmental factors. Bulletin of the Transilvania University of Braşov, 52(3), 83-90.

Jakschik, R., \& Pieniazeka, J. (2011). Über die Beweggründe für Abbrüche von zweitsprachlichen Alphabetisierungskursen und Anregungen für mögliche Gegenmaßnahmen. In Projektträger im Deutschen Zentrum für Luft- und Raumfahrt (Hrsg.), Lernprozesse in Alphabetisierung und Grundbildung Erwachsener: Diagnostik, Vermittlung, Professionalisierung. Bielefeld: wbv.

Käpplinger, B., Kulmus, C., \& Haberzeth, E. (2013). Weiterbildungsbeteiligung. Anforderungen an eine Arbeitsversicherung. WISO Diskurs. Bonn: Friedrich-Ebert-Stiftung. Expertise im Auftrag der Abteilung Wirtschafts- und Sozialpolitik der Friedrich-Ebert-Stiftung

Kelle, U., \& Kluge, S. (2010). Vom Einzelfall zum Typus. Fallvergleich und Fallkontrastierung in der qualitativen Sozialforschung. Wiesbaden: Springer VS.

Kluge, S. (1999). Empirisch begründete Typenbildung. Zur Konstruktion von Typen und Typologien in der qualitativen Sozialforschung. Wiesbaden: Springer VS.

Kramer, R.-T. (2017). „Habitus“ und „,kulturelle Passung“. In M. Rieger-Ladich, C. Grabau \& U.H. Bittlingmayer (Hrsg.), Pierre Bourdieu: Pädagogische Lektüren (S. 183-205). Wiesbaden: Springer VS. 
Kramer, R.-T., \& Helsper, W. (2010). Kulturelle Passung und Bildungsungleichheit - Potenziale einer an Bourdieu orientierten Analyse der Bildungsungleichheit. In H.-H. Krüger, U. Rabe-Kleberg, R.T. Kramer \& J. Budde (Hrsg.), Bildungsungleichheit revisited. Bildung und soziale Ungleichheit vom Kindergarten bis zur Hochschule (S. 103-125). Wiesbaden: Springer VS.

Kraus, K. (2015). Orte des Lernens als temporäre Konstellationen. Ein Beitrag zur Diskussion des Lernortkonzepts. In C. Bernhard, K. Kraus, S. Schreiber-Barsch \& R. Stang (Hrsg.), Erwachsenenbildung und Raum. Theoretische Perspektiven - professionelles Handeln - Rahmungen des Lernens (S. 41-53). Bielefeld: wbv.

Lingkost, A. (1996). Teilnahme und Nichtteilnahme an Funkkolleg-Begleitkursen. Eine qualitative Studie zur Drop-out-Forschung. Frankfurt a. M.: Deutsches Institut für Erwachsenenbildung.

McGivney, V. (2004). Understanding persistence in adult learning. Open Learning: The Journal of Open, Distance and E-Learning, 19(1), 33-46.

Meister, N., \& Sotzek, J. (2017). Habituelle Passungen und Nicht-Passungen angehender und berufseinsteigender Lehrpersonen im Sprechen über die (professionelle) schulische Praxis. In S. Lessenich (Hrsg.), Geschlossene Gesellschaften. Verhandlungen des 38. Kongresses der Deutschen Gesellschaft für Soziologie in Bamberg 2016.

Merkens, H. (1997). Stichproben bei qualitativen Studien. In B. Friebertshäuser \& A. Prengel (Hrsg.), Handbuch qualitative Forschungsmethoden in der Erziehungswissenschaft (S. 97-106). Weinheim: Juventa.

Meuser, M., \& Nagel, U. (2002). ExpertInneninterviews. Vielfach erprobt, wenig bedacht. In A. Bogner (Hrsg.), Das Experteninterview. Theorie, Methode, Anwendung (S. 71-93). Opladen: Leske + Budrich.

Mitscherlich, A. (1963). Auf dem Weg zur vaterlosen Gesellschaft. Ideen zur Sozialpsychologie. München: Piper.

Nuissl, E., \& Sutter, H. (1979). Dropout in der Weiterbildung. Eine Literaturexpertise empirischer Untersuchungen. Heidelberg: Esprint. Arbeitsgruppe für empirische Bildungsforschung

Park, J.-H., \& Choi, H. J. (2009). Factors influencing adult learners' decision to drop out or persist in online learning. Educational Technology \& Society, 12(4), 207-217.

Pickard, A. (2013). Attrition happens: Towards an acknowledgement and accommodation perspective of adult literacy student dropout. Journal of Research and Practice for Adult Literacy, Secondary, and Basic Education, 2(2), 114-126.

Przyborski, A., \& Wohlrab-Sahr, M. (2014). Qualitative Sozialforschung. Ein Arbeitsbuch (4. Aufl.). München: Oldenbourg.

Reich-Claassen, J. (2010). Warum Erwachsene (nicht) an Weiterbildungsveranstaltungen partizipieren. Einstellungen und prägende Bildungserfahrungen als Regulative des Weiterbildungsverhaltens. Berlin: LIT. Eine qualitativ-explorative Untersuchung erwartungswidriger Teilnahme und Nichtteilnahme an Erwachsenenbildung. (Zugl.: München, Univ. Diss. 2010)

Reich-Claassen, J. (2015). Weiterbildungsbeteiligung. In J. Dinkelaker \& A. von Hippel (Hrsg.), Erwachsenenbildung in Grundbegriffen (S. 75-84). Stuttgart: Kohlhammer.

Schäffter, O. (1992). Passung und Gegensteuerung. Das Zusammenspiel von Gegensätzen. In E. Nuissl (Hrsg.), Person und Sache. Zum 70. Geburtstag von Hans Tietgens (S. 173-181). Bad Heilbrunn: Klinkhardt.

Schmidt, B. (2011). Dropout in der Erwachsenenbildung. Zeitschrift für Pädagogik, 57(2), $203-213$.

Schmidt-Hertha, B., \& Tippelt, R. (2011). Typologien. REPORT-Zeitschrift für Weiterbildungsforschung, 1, 23-35.

Schrader, J. (1986). Teilnahmeverhalten und Teilnehmerschwund in Volkshochschulkursen. Frankfurt a. M.: Pädagogische Arbeitsstelle Deutscher Volkshochschul-Verband.

Schrader, J. (1994). Lerntypen bei Erwachsenen. Empirische Analysen zum Lernen und Lehren in der beruflichen Weiterbildung. Weinheim: Dt. Studien-Verlag. U.d.T.: Schrader, Josef: Lernstile in der beruflichen Weiterbildung. (Zugl.: Marburg, Univ. Diss. 1992)

Seitter, W. (Hrsg.). (2017). Zeit in der wissenschaftlichen Weiterbildung. Wiesbaden: Springer VS.

Shank, J.A., \& McCracken, J.D. (1993). Dropout and completion in adult vocational job training programs. A prediction model for the adult vocational student. Paper presented at 1993 American Vocational Association

Stamm, M., Holzinger-Neulinger, M., \& Suter, P. (2012). Schulabbrecher in unserem Bildungssystem. Wiesbaden: Springer VS.

Strauss, A., \& Corbin, J. (1996). Grounded Theory. Grundlagen Qualitativer Sozialforschung. Weinheim: Beltz. 
Ten Dam, G. T. M. (1995). Drop-out from adult education. Social environment, school culture and perceptions. International Journal of Lifelong Education, 14(1), 51-63.

Tietgens, H. (1981). Die Erwachsenenbildung. München: Juventa.

Tietgens, H. (1982). Angebotsplanung. In E. Nuissl (Hrsg.), Taschenbuch der Erwachsenenbildung (S. 122-144). Baltmannsweiler: Pädagogischer Verlag Burgbücherei.

Tinto, V. (1975). Dropout from higher education. A theoretical synthesis of recent research. Review of Educational Research, 45(1), 89-125.

Tinto, V. (1993). Leaving college. Rethinking the causes and cures of student attrition. Chicago: University of Chicago Press.

Vontobel, J. (1972). Über den Erfolg in der Erwachsenenbildung. Empirische Basisstudie zum Problem der Erfassung des Bildungserfolgs. Braunschweig: Westermann.

Witzel, A. (2000). Das problemzentrierte Interview. Forum Qualitative Sozialforschung. https://doi.org/10. 17169/fqs-1.1.1132.

Publisher's Note Springer Nature remains neutral with regard to jurisdictional claims in published maps and institutional affiliations. 